Published in final edited form as:

J Biomech Eng. 2004 August ; 126(4): 523-528.

\title{
HARP MRI Tagging for Direct Quantification of Lagrangian Strain in Rat Hearts After Myocardial Infarction
}

\author{
Wei Liu ${ }^{1,2}$, Junjie Chen ${ }^{1,2}$, Songbai $\mathrm{Ji}^{3}$, J. Stacy Allen ${ }^{1}$, Philip V. Bayly ${ }^{3}$, Samuel A. \\ Wickline $^{1,2}$, and $\mathrm{Xin} \mathbf{Y u}^{1,2, *}$ \\ 1Cardiovascular MR Laboratories, Cardiovascular Division, Washington University School of Medicine \\ 2Department of Biomedical Engineering, Washington University in St. Louis, St. Louis, MO USA \\ 3Department of Mechanical Engineering, Washington University in St. Louis, St. Louis, MO USA
}

\begin{abstract}
The utility of harmonic phase (HARP) analysis has recently been demonstrated in humans and large animals as a technique for rapid and automatic analysis of tagged MR images. In the current study, the applicability and accuracy of HARP analysis for automatic strain quantification in small animals were investigated. A validation study was performed on seven post-infarct rats and seven agematched controls. A method for direct computation of 2D Lagrangian strain fields from spatial derivatives of HARP images was also developed in this paper. The results of HARP analysis were evaluated by comparison with those of homogeneous strain analysis employing finite element method and manual tag tracking. Both methods were validated with simulated digital images. Compared to conventional homogeneous strain analysis, HARP analysis yielded similar results in the assessment of regional strain patterns in both control and infarct rats. Both methods detected a reduction in maximal stretch and shortening in infarct rats. Our results suggest that HARP analysis can also be applied to quantify alterations in regional myocardial wall motion in small animals.
\end{abstract}

\section{Keywords}

MR Tagging; HARP; myocardial strain; myocardial infarction

\section{INTRODUCTION}

Magnetic Resonance (MR) tagging allows comprehensive and noninvasive assessment of regional myocardial contractile performance [1]. The spatial modulation of magnetization (SPAMM) technique uses gradient and RF pulses to generate parallel dark bands within an image $[2,3]$. These bands deform with the myocardium during the cardiac cycle and can be used to quantitatively evaluate regional myocardial strain fields. Over the last decade, numerous studies using this technique have contributed to our understanding of cardiac biomechanics in both normal and diseased hearts [4-12]. The utility of MR tagging in assessing cardiac function of small animals such as rats and mice was also demonstrated recently [13-16]. However, current methods of manual or semi-automatic image analysis require substantial user input and processing time. Such time-consuming post-processing is not suitable for large-scale studies or routine clinical use.

\footnotetext{
*Address correspondence to: Xin Yu, Sc.D. Campus Box 8086660 S. Euclid Ave. St. Louis, MO 63110 Telephone: 314-454-7410 Fax: 314-454-7490 Email: xin@cvu.wustl.edu.
} 
Harmonic phase (HARP) analysis has recently been proposed as a technique for rapid and automatic strain quantification [17-19]. The technique is based on the fact that tissue motion is directly encoded in HARP images of the off-center spectral peaks in the Fourier domain of SPAMM tagged images. Osman et al. developed the method to directly calculate the 2D Eulerian strains from spatial derivatives of HARP images. The accuracy and sensitivity of such analysis were evaluated in humans and large animals [20,21]. However, the applicability of HARP analysis in small animals has not been investigated.

Rodent models, especially rats and mice, have assumed an increasingly prominent role in cardiovascular research. The development of rapid, noninvasive methods for characterizing ventricular myofiber motion in these animals can provide new opportunities to elucidate the molecular mechanisms of myocardial function. However, the application of HARP analysis to small animals presents additional challenges as compared with its use in humans and larger animals. In addition to the difficulties encountered in cardiac triggering associated with higher heart rates and shorter QRS durations in small animals, the use of larger gradients to achieve higher imaging/tagging resolution may also compromise SNR. Because the calculation of spatial derivatives of HARP images depends critically on the SNR of these images, the applicability of such methods to small animal imaging requires careful evaluation.

Alternatively, it was demonstrated by Kuijer et al. that the use of 1-1 CSPAMM led to improved myocardial strain maps with HARP analysis [22]. Such improvement was achieved through the suppression of the central peak, which is the primary source of artifacts in calculated strain maps. In human studies, successful suppression of the central peak was accomplished through breath-holding, which is difficult to perform in small animals. In a recent study, Gilson et al reported partial suppression of the central peak in mice with complementary DENSE technique without breath-holding [23]. Better peak suppression may be accomplished with respiratory gating [24]. However, the significantly higher heart rate renders the fast and single-shot imaging techniques impractical in small animals. The acquisition of two complementary data sets with respiratory gating will significantly increase the already prolonged image acquisition time.

The purpose of the current study was to investigate the accuracy of HARP analysis for fast quantification of myocardial strain fields in small animals under practical situations. We sought to demonstrate the sensitivity of HARP analysis in detecting alterations in ventricular wall motion in a rat model of myocardial infarction. We also implemented a method for direct computation of Lagrangian strain fields from the spatial derivatives of HARP images. The results of the HARP analysis were validated by comparison with those of homogeneous strain analysis that employed finite element method and traditional manual tag tracking.

\section{MATERIALS AND METHODS}

\section{Animal Preparation}

20-24 month old Fischer 344 rats $(n=7)$ underwent thoracotomy and permanent ligation of the left anterior descending artery (LAD). Additional sham-infarct procedures (thoracotomy and pericardial incision) were performed in age-matched rats $(n=7)$ that served as the controls. Briefly, the animal was anesthetized, intubated, and ventilated with supplemental oxygen. Via a lateral thoracotomy, the proximal LAD was permanently occluded with a surgical suture. Rats were treated with buprenorphine for pain relief and maintained under physiological conditions in a pathogen free environment for four weeks. All the procedures conformed to the guidelines set forth by Washington University. 


\section{MRI Imaging}

MRI experiments were performed four weeks after surgery on a 4.7T Varian INOVA system (Varian Associates, Palo Alto, CA) equipped with a gradient insert $(60 \mathrm{G} / \mathrm{cm}, 10 \mathrm{~cm}$ inner diameter). A $5 \mathrm{~cm}$ surface coil was built for image acquisition. Animals were anesthetized with $1 \%$ isoflurane by nose cone and placed into the coil in prone position. Electrodes were attached to front paws and right leg for ECG gating and monitoring of vital signs. The animals were kept warm by blowing hot air into the magnet using a blow dryer. The heat flow and the anesthesia level were manually adjusted to maintain the heart rate at $260 \sim 320$ beats $/ \mathrm{min}$. A horizontal long-axis view (four-chamber view) was acquired perpendicular to the interventricular septum. Three short-axis slices, parallel to the tricuspid and mitral valve plane, were imaged at basal, midventricular, and apical levels. The midventricular slice was chosen at $50 \%$ of the distance between the atrioventricular valve plane and the apex at diastole. Basal and apical slices were chosen $3 \mathrm{~mm}$ above and below the midventricular slice, respectively.

MR tags were generated by applying the tagging sequence immediately after the detection of the R-wave. Two sets of SPAMM1331 pulses were executed sequentially to generate stripe tags in two orthogonal directions in one acquisition series. The tagging gradient was 10 Gauss/ $\mathrm{cm}$ with a pulse delay of $0.2 \mathrm{~ms}$. These parameters yielded a tag grid spacing of $0.9 \mathrm{~mm}$. Immediately following the tagging sequences, gradient-echo cine images were acquired during the entire cardiac cycle to follow the displacement of the tags. Repetition time (TR) was adjusted according to the R-R interval of the heart such that 15 frames were acquired during one cardiac cycle. Other imaging parameters were: flip angle, $30^{\circ}$; echo time (TE), $3 \mathrm{~ms}$; data matrix, 256×256; field of view (FOV), $6.5 \mathrm{~cm} \times 6.5 \mathrm{~cm}$; slice thickness, $1.5 \mathrm{~mm}$. The k-space data were zero-filled into a $512 \times 512$ data matrix, yielding an in-plane resolution of $127 \mu \mathrm{m} \times 127$ $\mu \mathrm{m}$. Regular gradient-echo cine images provided better contrast between myocardium and blood and were acquired for the purposes of myocardial contour tracing and calculation of ejection fraction. These images were acquired at the same time points as the tagged cine images using the following parameters: FOV, $6.5 \mathrm{~cm} \times 6.5 \mathrm{~cm}$; data matrix, $128 \times 128$; slice thickness, $1.5 \mathrm{~mm}$. Non-tagged images were also zero-filled into a $512 \times 512$ data matrix so that myocardial contours traced from these images were directly used in the analysis of tagged images with minimal adjustment.

\section{Data Analysis}

MR images were transferred to a PC for data analysis. A MATLAB-based (MathWorks, Natick, MA) Cardiovascular MR Image Analysis Tool (CVMRI) was developed for image analysis. Epicardial and endocardial contours were traced interactively in each temporal frame from regular cine images. Contour tracing used the B-spline method with eight control points. The traced contours were stacked to compute ventricular volume and ejection fraction.

HARP analysis-The SPAMM modulation of an MR image takes the form of a sinusoidal dependence of the longitudinal magnetization, which produces an array of spectral peaks (harmonic peaks) in the Fourier domain (k-space, Figure 1A). The inverse Fourier transform of a harmonic peak has two parts. The magnitude image of a harmonic peak reflects the anatomy of the heart (Figure 1B). HARP image refers to the corresponding phase image of a harmonic peak (Figure 1C). The phase angle is a material property of the tagged tissue. It remains invariant throughout the cardiac cycle for a particular material point. Hence, the phase angle can be used to track the motion of the underlying tissue (Figure 1D). It was shown by Osman et al. that the displacement field is encoded in a HARP image and can be used for direct strain calculation [17-19], that is,

$$
\frac{\partial \mathbf{q}}{\partial \mathbf{y}}=\left(W^{T} H\right)^{-1} \frac{\partial \phi}{\partial \mathbf{y}}
$$


where $\mathbf{q}$ is the position of a material point in the reference map at time $t=0, \mathbf{y}(\mathbf{q}, t)$ is the position of the same material point at time $t$ as shown in Figure 2, $\varphi$ is a vector describing the harmonic phases in the two tagging directions, and $W$ and $H$ are the matrices associated with the tagging and imaging planes [18].

Based on this property, Osman et al. developed a method for direct calculation of the strain in a given direction [18]. We now propose a method for calculating Lagrangian strains to provide a direct comparison with the conventional homogeneous strain analysis. The 2D Lagrangian strain tensor can be calculated as

$$
\mathbf{E}=\frac{1}{2}\left(\mathbf{F}^{T} \mathbf{F}-\mathbf{I}\right)
$$

where $\mathbf{F}=\frac{\partial y}{\partial q}$ is the $2 \mathrm{D}$ deformation gradient tensor referred to undeformed state at time $t=0$.

$$
\begin{aligned}
& \text { Since }^{\frac{\partial y}{\partial q}}=\left(\frac{\partial q}{\partial y}\right)^{-1} \text {, from Eq. (1) we have } \\
& \mathbf{F}=\left(\frac{\partial \phi}{\partial \mathbf{y}}\right)^{-1} W^{T} H
\end{aligned}
$$

Therefore, the 2D Lagrangian strain tensor can be calculated as

$$
\mathbf{E}=\frac{1}{2}\left(H^{T} W\left(\frac{\partial \phi}{\partial \mathbf{y}}\right)^{-T} \cdot\left(\frac{\partial \phi}{\partial \mathbf{y}}\right)^{-1} W^{T} H-\mathbf{I}\right)
$$

In the present study, harmonic peaks of a tagged image were reconstructed from the Fourier transform of the corresponding magnitude image to remove phase variations not associated with myocardial motion. A circular band-pass filter was used to extract the off-center harmonic peaks. The radius of the filter was chosen at half of the tagging frequency. The spatial derivatives of the harmonic phases were computed from unwrapped HARP angle images using method proposed by Osman et al. [18]. The calculated Lagrangian strain tensor $\mathbf{E}$ was further diagonalized to yield two principal strains $\left(E_{1}\right.$ and $\left.E_{2}\right)$ independent of any coordinate system. $E_{1}$ and $E_{2}$ were defined as the most positive (maximal stretch) and most negative strains (maximal shortening) respectively.

Homogeneous Strain Analysis-Conventional homogeneous strain analysis was performed to provide comparison with HARP analysis. Myocardial contour was traced using B-spline curve with 8 control points (Figure 1E). The tagging mesh was tracked interactively using coupled cubic spline snakes (Figure 1F) [25]. These snakes were constructed by having the horizontal and vertical lines share the control points such that horizontal and vertical lines would both deform to fit the corresponding tag lines when a control point shared by these two lines was moved. Following tag tracking, myocardium was divided into non-overlapping triangular tissue elements using sets of adjacent tag points as the vertices (Figure 1G). Regional myocardial wall motion across the short-axis plane of the ventricle was visualized by tracking the displacement of the centroid of each triangle (Figure 1H). Lagrangian strain tensor $\mathbf{E}$ was computed for each triangle using method outlined by Fogel et al. [26]. This approach assumes that deformation within each triangle is locally homogeneous. $\mathbf{E}$ was also diagonalized to provide direct comparison of the principal strains with those calculated from HARP analysis.

\section{Histological Analysis}

Upon the completion of MR examination, the animal was sacrificed, and the heart excised, weighed, and fixed in neutral-buffered $10 \%$ formalin for histological analysis. The fixed heart was sliced in $2 \mathrm{~mm}$ increment from base to apex along the left ventricular (LV) long-axis. Each slice was embedded in paraffin and sectioned at $4 \mu \mathrm{m}$. The tissue sections were stained with 
Masson's trichrome for the identification of scar tissue. Infarct size was calculated using the method described previously [27].

\section{Statistical Analysis}

All measurements are presented as mean \pm SD. Mean values of myocardial strains in infarct and control groups were compared by unpaired Student's $t$-test. Comparisons between HARP and homogeneous strain analysis were assessed using linear regression analysis and Bland-Altman plots. $p$ values less than 0.05 were considered statistically significant.

\section{RESULTS}

\section{Validation of Methods}

To validate the strain calculation in both methods, simulated tagging images were constructed by applying sinusoidal modulation to a digital circular image. A circular band-pass filter was used to extract the off-center harmonic peak. The radius of the filter was chosen at half of the tagging frequency. Stretch strains ranging from 0.1 to 0.8 and shear strains ranging from 0.05 to 0.3 were applied to the original images to induce deformation (Figure 3A-C). 2D strain tensor was calculated using both HARP and homogeneous strain analysis. The calculated strains showed good agreement with theoretical values in both methods (Figure 3F-G). To evaluate the noise response of the two methods, Gaussian noise ranging from $20 \%$ to $100 \%$ was added to the digital images (Figure 3D-E). The calculated strains showed good agreement with the true values, suggesting that both methods are insensitive to Gaussian noise (Figure $3 \mathrm{H}-\mathrm{I})$.

\section{Physiologic Data}

The mean heart rate during the MRI study was $278 \pm 21$ beats/min in the control group and 287 \pm 29 beats/min in the infarct group ( $\mathrm{p}=$ N.S.). Significant $\mathrm{LV}$ dilation occurred in infarct group. End-diastolic volume was $0.61 \pm 0.08 \mathrm{~mL}$ in infarct group and $0.50 \pm 0.11 \mathrm{~mL}$ in control group $(p<0.05)$. End-systolic volume in infarct rats was also larger: $0.25 \pm 0.07 \mathrm{~mL}$ in infarct group and $0.15 \pm 0.04 \mathrm{~mL}$ in control group $(p<0.005)$. The ejection fraction was significantly reduced in infarct rats as compared to controls $(58.7 \pm 6.7 \%$ versus $70.0 \pm 4.8 \%, p<0.001)$. Histological analysis confirmed that infarction occurred in the anterior-lateral regions of the heart at the apical level. Average infarct size was $14.8 \pm 5.0 \%$ of the total LV mass.

\section{Strain Analysis}

Typical postprocessing time with homogeneous strain analysis was 4 hours for each heart, while that for HARP analysis was only about $10 \mathrm{~min}$. Good agreement was observed for strain calculations using both methods as demonstrated by the color maps of $E_{1}$ and $E_{2}$ strains from both infarct and control hearts (Figure 4). Quantitatively, no significant differences in strain calculation by the two methods were detected in both control and infarct groups. Both methods detected reduced magnitude of $E_{1}$ and $E_{2}$ strains after myocardial infarction (Figure 5 and Table 1). At the apex where infarction occurred, maximal stretch (strain $E_{1}$ ) was significantly decreased in the infarct group. The magnitude of maximal shortening $\left(\right.$ strain $\left.\mathrm{E}_{2}\right)$ in the infarct group was also reduced throughout the entire ventricle. Maximal stretch in noninfarct regions (base and midventricle) was the same as that of the controls by both methods.

For pooled data from both control and infarct groups, the two methods exhibited good correlation for assessment of myocardial strains. Quantitative comparisons using linear regression analysis are shown in Figure 6. The correlation coefficients were 0.95 for strain $E_{1}$ and 0.92 for strain $E_{2}(p<0.0001)$. Comparisons between data are also displayed in Bland- 
Altman plots in Figure 7. Mean differences between the two methods were $0.008 \pm 0.038$ for $\mathrm{E}_{1}$ and $0.007 \pm 0.010$ for $\mathrm{E}_{2}(\mathrm{p}=\mathrm{N}$.S.).

To further evaluate the accuracy of HARP analysis on regional wall motion, each slice was segmented into septal, inferior, lateral and anterior regions. Figure 8 shows regional apical strains calculated from both methods. Both methods detected a reduction in $\mathrm{E}_{1}$ strain in infarct rats in all the four segments (Figure 8A). The magnitude of $\mathrm{E}_{2}$ strain calculated from both methods was also significantly reduced in the inferior, lateral and anterior regions, but remained the same as that of the control rats in the septum (Figure 8B). Segmented data at midventricular and basal slices also demonstrated similar reduction in $\mathrm{E}_{2}$ strain in the lateral and anterior regions. Calculated strains in segmented regions from all three slices exhibited good correlation between HARP analysis and homogeneous method (Figure 9). The correlation coefficients were 0.91 for strain $\mathrm{E}_{1}$ and 0.84 for strain $\mathrm{E}_{2}$, respectively.

\section{DISCUSSION}

We validated the utility of HARP analysis for rapid quantification of myocardial strain fields from SPAMM-tagged MR images of rat hearts. Our results demonstrate that measurements of 2D myocardial strains by HARP agree well with those obtained by conventional homogeneous strain analysis based on manual tracking of tag lines. The two methods yielded similar results in the assessment of regional strain patterns in both control and infarct rats. Both methods detected a reduction in maximal stretch and shortening in infarct rats.

The original strain quantification proposed by Osman et al. was based on the calculation of Eulerian strain in a given direction [18]. We implemented a HARP analysis method for direct calculation of 2D Lagrangian strains in the current study. Because the Lagrangian strain tensor is widely used in classical continuum mechanics and in many tagging and non-tagging studies, direct calculation of Lagrangian strain tensor should allow more straightforward comparisons among different laboratories.

Validation study performed on a digital phantom showed strong agreement between the calculated strains using both methods and the theoretical values. Noise analysis revealed that both methods were insensitive to Gaussian noise. Such results are expected for homogenous approach with manual tag tracing. The robust noise response in HARP analysis is due to the fact that the filter used to extract the harmonic peak covers only a small fraction of the k-space. Therefore, it also serves as a noise filter. As a result, a large portion of the noise is suppressed in HARP analysis.

To date, most MRI tagging studies have used the homogeneous methods to calculate myocardial strain fields $[7,9,26]$. The homogeneous methods also have been used in nontagging studies that employed metallic bead implantation to evaluate myocardial deformation [28]. Frequently, the first frame is taken as the reference frame, i.e. the undeformed frame. Therefore, it is very important that the first frame is acquired during isovolumic contraction when myocardial wall motion is minimal. In small animal imaging, this presents a challenge because the duration of the QRS complex is only about $20 \mathrm{~ms}$. In addition, the requirement of higher tagging resolution can only be achieved with either larger tagging gradients or longer pulse delays, which can prolong the implementation time of the tagging sequence. In the current study, the tagging-time was $7 \mathrm{~ms}$ and the echo-time (TE) for gradient-echo cine imaging was $3 \mathrm{~ms}$. Therefore, deformation of tag lines in the reference frame was negligible, especially when myocardial strains were large. On the contrary, HARP analysis does not require a reference frame. Strains are calculated directly from the spatial derivatives of the deformed HARP images, based on the assumption that at time $t=0$, isophase contours, or tag lines, are 
undeformed. Therefore, it will not affect the accuracy of strain calculation whether the first frame is acquired within the QRS complex or not.

Linear regression was performed in the current study to evaluate the correlation between the two methods. Because linear regression calculates the best fit to the data, the slopes of the linear regression for both $E_{1}$ and $E_{2}$ strains were less than one with nonzero intercepts. If we force the intercepts in the linear regression to be zero, the correlation would be $y=0.95 \times$ for $E_{1}$ and $y=0.99$ for $E_{2}$ with a correlation coefficient of 0.91 for both strains. These nonzero intercepts suggest that for small strain values, strains calculated from HARP are larger in magnitude than those calculated from homogeneous analysis. It is not completely clear to us what caused this discrepancy at small strain values. One possibility is, as was discussed above, that the references for the two methods differ slightly. If the first frame in the image series is slightly deformed, homogeneous analysis may underestimate the strain, especially when the magnitudes of the strains are small.

Although not statistically significant, the $\mathrm{E}_{1}$ Bland-Altman curve showed a trend of bias when strain values are large, suggesting that HARP analysis may have underestimated large strains. Such bias was also present in a human study involving postinfarct patients [20]. It is possible that the band-pass filter used to extract the harmonic peaks have suppressed the tag modulation in areas with large strains, leading to the underestimation of the strains in these areas.

SPMM1331 tagging sequences were used in the current study to generate sharper tag lines to facilitate manual tag tracing for homogeneous strain analysis, because inaccuracy in tag tracing also can introduce errors in strain calculation. In theory, the longitudinal magnetization from SPAMM1331 does not take the form of a simple sinusoidal dependence. Instead, it takes the form of sinusoidal square [29], which leads to an array of spectral peaks in k-space. However, for each individual harmonic peak, the theory that describes the dependence of HARP images on the displacement field of the myocardium still holds. Besides, the high tagging resolution required by small animal imaging resulted in greater separation of the harmonic peaks in kspace. Therefore, interference from neighboring harmonic peaks was minimal. Our results demonstrated a very good correlation between the two methods, suggesting that errors caused by using SPAMM1331 sequence were minimal in HARP analysis.

In a recent study, Gilson et al. reported the quantification of myocardial strains in post-infarct mice using complementary DENSE technique [23]. The study shows that strains measured by DENSE and tagging methods are in good agreement. Current image acquisition is more efficient for tagging MRI. The typical acquisition time for one data set that covered the whole cardiac cycle (15 frames) was about 10 min, while the scan time for a complete DENSE data set at end-systole was $35 \mathrm{~min}$. Our recent study suggests that satisfactory accuracy can be achieved with partial k-space sampling [33]. Such technique will further reduce the scan time to 3-4 min.

In the current study, both HARP and homogeneous analysis delineated the reduction of maximal stretch $\left(\mathrm{E}_{1}\right)$ only in the infarct region. In contrast, maximal shortening $\left(\mathrm{E}_{2}\right)$ was decreased throughout the entire ventricle. Similar functional alterations have been observed in humans [30]. Kramer and colleagues also reported decreased intramyocardial circumferential shortening throughout LV in both animals and patients [31,32]. However, it is not completely understood what mechanisms are responsible for functional alterations in remote viable myocardium. MR tagging on genetically manipulated mouse models may help our investigation of the underlying mechanisms that govern the functional adaptations in noninfarct regions.

Although the current study was performed on rat hearts, our previous tagging studies on mice suggest that it is possible to achieve higher SNR in tagged images of mouse hearts [34,35]. 
Since a mouse heart is smaller and closer to the chest wall, a smaller surface coil can be used for image acquisition which will further enhance the SNR. The applicability of HARP analysis in mouse imaging will greatly facility the investigation of functional phenotypes of genetically manipulated mouse models of cardiovascular disease. Such an approach may ultimately prove to be of use for dissecting molecular mechanisms responsible for altered contractile behavior in diseased hearts.

\section{ACKNOWLEDGEMENTS}

The authors wish to thank Dr. Joseph J.H. Ackerman, Director of the Biomedical MR Laboratory (BMRL) at Washington University, for the advice and MR resources. The authors are indebted to Dr. Sheng-Kwei Song, who provided invaluable technical assistance. The authors acknowledge the support of the Washington University Small Animal Imaging Resource (WUSAIR) funded in part through NCI Small Animal Imaging Research Program (SAIRP) grant R24 CA83060. This work also was supported by NIH grants R01 HL73315 (X.Y.), R01 HL42950 (S.A.W.). Wei Liu was supported by a predoctoral fellowship from the Heartland Affiliate of the American Heart Association (0215174Z). The authors thank Dr. Andrew Bowman for critical review of the manuscript.

\section{References}

1. Zerhouni EA, Parish DM, Rogers WJ, Yang A, Shapiro EP. Human heart: tagging with MR imaging-a method for noninvasive assessment of myocardial motion. Radiology 1988;169:59-63. [PubMed: 3420283]

2. Axel L, Dougherty L. Heart wall motion: improved method of spatial modulation of magnetization for MR imaging. Radiology 1989;172:349-350. [PubMed: 2748813]

3. Axel L, Dougherty L. MR imaging of motion with spatial modulation of magnetization. Radiology 1989;171:841-845. [PubMed: 2717762]

4. McVeigh ER, Zerhouni EA. Noninvasive measurement of transmural gradients in myocardial strain with MR imaging. Radiology 1991;180:677-683. [PubMed: 1871278]

5. Rademakers FE, Rogers WJ, Guier WH, Hutchins GM, Siu CO, Weisfeldt ML, Weiss JL, Shapiro EP. Relation of regional cross-fiber shortening to wall thickening in the intact heart. Three-dimensional strain analysis by NMR tagging. Circulation 1994;89:1174-1182. [PubMed: 8124804]

6. Moore CC, McVeigh ER, Zerhouni EA. Quantitative tagged magnetic resonance imaging of the normal human left ventricle. Top Magn Reson Imaging 2000;11:359-371. [PubMed: 11153703]

7. Fogel MA, Weinberg PM, Hubbard A, Haselgrove J. Diastolic biomechanics in normal infants utilizing MRI tissue tagging. Circulation 2000;102:218-224. [PubMed: 10889134]

8. Young AA, Kramer CM, Ferrari VA, Axel L, Reichek N. Three-dimensional left ventricular deformation in hypertrophic cardiomyopathy. Circulation 1994;90:854-867. [PubMed: 8044957]

9. Azhari H, Weiss JL, Rogers WJ, Siu CO, Shapiro EP. A noninvasive comparative study of myocardial strains in ischemic canine hearts using tagged MRI in 3-D. Am J Physiol 1995;268:H1918-1926. [PubMed: 7771541]

10. Lima JA, Ferrari VA, Reichek N, Kramer CM, Palmon L, Llaneras MR, Tallant B, Young AA, Axel L. Segmental motion and deformation of transmurally infarcted myocardium in acute postinfarct period. Am J Physiol 1995;268:H1304-1312. [PubMed: 7900884]

11. Stuber M, Scheidegger MB, Fischer SE, Nagel E, Steinemann F, Hess OM, Boesiger P. Alterations in the local myocardial motion pattern in patients suffering from pressure overload due to aortic stenosis. Circulation 1999;100:361-368. [PubMed: 10421595]

12. Young AA, Dokos S, Powell KA, Sturm B, McCulloch AD, Starling RC, McCarthy PM, White RD. Regional heterogeneity of function in nonischemic dilated cardiomyopathy. Cardiovasc Res 2001;49:308-318. [PubMed: 11164841]

13. de Crespigny AJ, Carpenter TA, Hall LD. Cardiac tagging in the rat using a DANTE sequence. Magn Reson Med 1991;21:151-156. [PubMed: 1943673]

14. Henson RE, Song SK, Pastorek JS, Ackerman JJ, Lorenz CH. Left ventricular torsion is equal in mice and humans. Am J Physiol 2000;278:H1117-1123.

15. Epstein FH, Yang Z, Gilson WD, Berr SS, Kramer CM, French BA. MR tagging early after myocardial infarction in mice demonstrates contractile dysfunction in adjacent and remote regions. Magn Reson Med 2002;48:399-403. [PubMed: 12210951] 
16. Zhou R, Pickup S, Glickson JD, Scott CH, Ferrari VA. Assessment of global and regional myocardial function in the mouse using cine and tagged MRI. Magn Reson Med 2003;49:760-764. [PubMed: 12652548]

17. Osman NF, Kerwin WS, McVeigh ER, Prince JL. Cardiac motion tracking using CINE harmonic phase (HARP) magnetic resonance imaging. Magn Reson Med 1999;42:1048-1060. [PubMed: 10571926]

18. Osman NF, McVeigh ER, Prince JL. Imaging heart motion using harmonic phase MRI. IEEE Trans Med Imaging 2000;19:186-202. [PubMed: 10875703]

19. Osman NF, Prince JL. Visualizing myocardial function using HARP MRI. Phys Med Biol 2000;45:1665-1682. [PubMed: 10870717]

20. Garot J, Bluemke DA, Osman NF, Rochitte CE, McVeigh ER, Zerhouni EA, Prince JL, Lima JA. Fast determination of regional myocardial strain fields from tagged cardiac images using harmonic phase MRI. Circulation 2000;101:981-988. [PubMed: 10704164]

21. Garot J, Bluemke DA, Osman NF, Rochitte CE, Zerhouni EA, Prince JL, Lima JA. Transmural contractile reserve after reperfused myocardial infarction in dogs. J Am Coll Cardiol 2000;36:2339_ 2346. [PubMed: 11127482]

22. Kuijer JP, Jansen E, Marcus JT, van Rossum AC, Heethaar RM. Improved harmonic phase myocardial strain maps. Magn Reson Med 2001;46:993-999. [PubMed: 11675652]

23. Gilson WD, Yang Z, French BA, Epstein FH. Complementary displacement-encoded MRI for contrast-enhanced infarct detection and quantification of myocardial function in mice. Magn Reson Med 2004;51:744-752. [PubMed: 15065247]

24. Schneider, JE.; Cassidy, PJ.; Lygate, C.; Tyler, DJ.; Clarke, K.; Neubauer, S. Proc ISMRM. Toronto, Canada: 2003. High-resolution, cine-imaging of mouse hearts at $12 \mathrm{~T}$ in vertical position- a challenge.; p. 1553

25. Amini AA, Chen Y, Curwen RW, Mani V, Sun J. Coupled B-snake grids and constrained thin-plate splines for analysis of 2D tissue deformation from tagged MRI. IEEE Trans Med Imaging 1998;17:344-356. [PubMed: 9735898]

26. Fogel MA, Krishanu B, Weinberg PM, Hoffman EA. Regional wall motion and strain analysis across stages of Fontan reconstruction by magnetic resonance tagging. Am J Physiol 1995;269:H11311152.

27. Chen J, Song SK, Liu W, McLean M, Allen JS, Tan J, Wickline SA, Yu X. Remodeling of cardiac fiber structure after infarction in rats quantified with diffusion tensor MRI. Am J Physiol 2003;285:H946-954.

28. Waldman LK, Fung YC, Covell JW. Transmural myocardial deformation in the canine left ventricle. Normal in vivo three-dimensional finite strains. Circ Res 1985;57:152-163. [PubMed: 4006099]

29. Hore PJ. Solvent suppression in Fourier transform nuclear magnetic resonance. J Magn Reson 1983;55:283-300.

30. Bogaert J, Bosmans H, Maes A, Suetens P, Marchal G, Rademakers FE. Remote myocardial dysfunction after acute anterior myocardial infarction: impact of left ventricular shape on regional function: a magnetic resonance myocardial tagging study. J Am Coll Cardiol 2000;35:1525-1534. [PubMed: 10807456]

31. Kramer CM, Lima JA, Reichek N, Ferrari VA, Llaneras MR, Palmon LC, Yeh IT, Tallant B, Axel L. Regional differences in function within noninfarcted myocardium during left ventricular remodeling. Circulation 1993;88:1279-1288. [PubMed: 8353890]

32. Kramer CM, Rogers WJ, Theobald TM, Power TP, Petruolo S, Reichek N. Remote noninfarcted region dysfunction soon after first anterior myocardial infarction. A magnetic resonance tagging study. Circulation 1996;94:660-666. [PubMed: 8772685]

33. Liu, W.; Chen, J.; Allen, JS.; Wickline, SA.; Yu, X. Proc ISMRM. Kotyo, Japan: 2004. Fast imaging of cardiac strain using partial k-space HARP in mice.; p. 1790

34. Yu, X.; Liu, W.; Chen, J.; Lin, SJ.; McLean, MD.; Allen, JS.; Grady, RM.; Wickline, SA. Proc ISMRM. Honolulu, Hawaii: 2002. MRI tagging revealed reduced ventricular torsion in muscular dystrophic mice.; p. 1691

35. Liu, W.; Chen, J.; Wickline, SA.; Grady, RM.; Yu, X. Proc ISMRM. Kyoto, Japan: 2004. Alterations in left ventricular contractility in dystrobrevin-knockout mice characterized by MR tagging.; p. 1791 

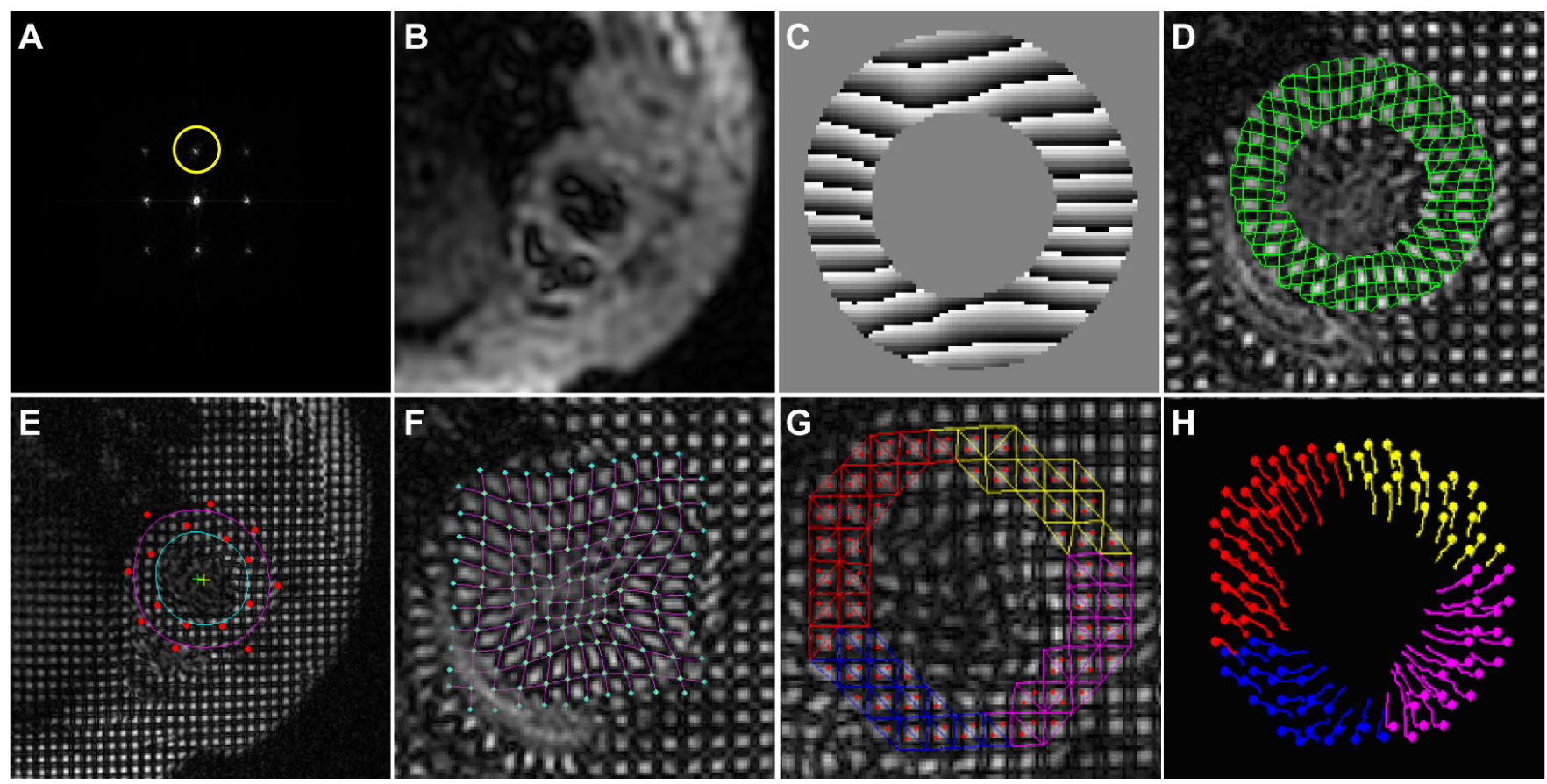

Figure 1.

Representative images and data analysis. A. SPAMM-tagged image in k-space. Yellow circle represents one filter used for extracting the harmonic peak. B. The corresponding magnitude image of the harmonic peak. C. The corresponding HARP image masked by myocardial contours. D. Synthetic tag lines generated from the isophase contours of $\pi / 2$. E. Manually traced myocardial contours using B-spline method. F. SPAMM-tagged image overlaid with manually traced tag lines and intersecting tag points. G. Triangulation of the myocardium for homogeneous strain analysis. Each red dot represents the centroid of a triangle. H. Visualization of regional myocardial wall motion with the trajectories of the centroids from diastole to systole. Yellow, red, blue, and magenta colors represent lateral, inferior, septal, and anterior regions, respectively. 


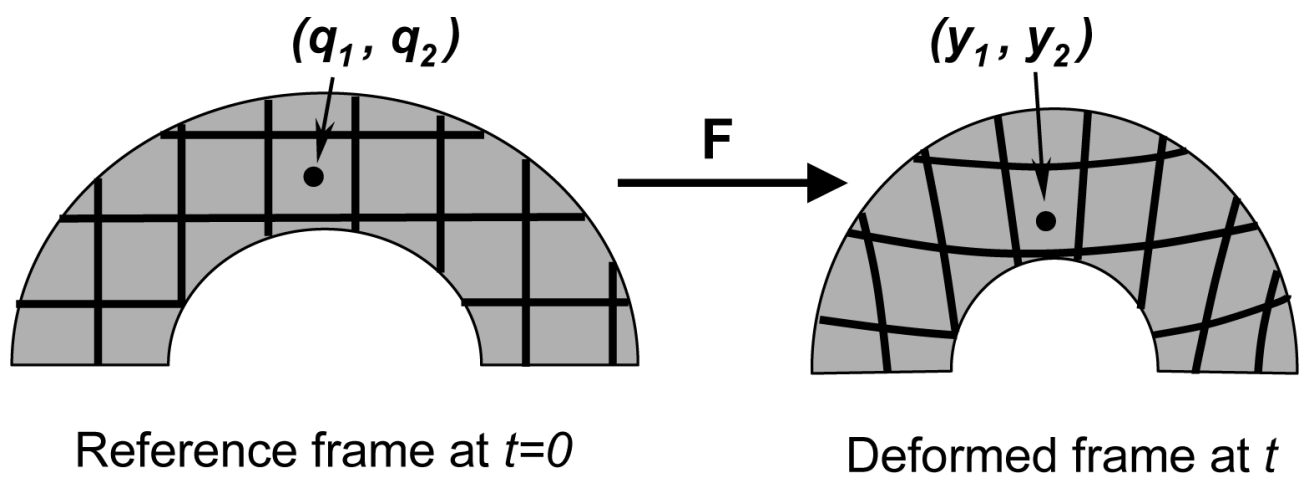

Figure 2.

Schematic illustration of a material point in initial reference frame at time $t=0(\mathbf{q})$ and deformed frame at time $t(\mathbf{y})$. $\mathbf{F}$ is the $2 \mathrm{D}$ deformation gradient tensor. 

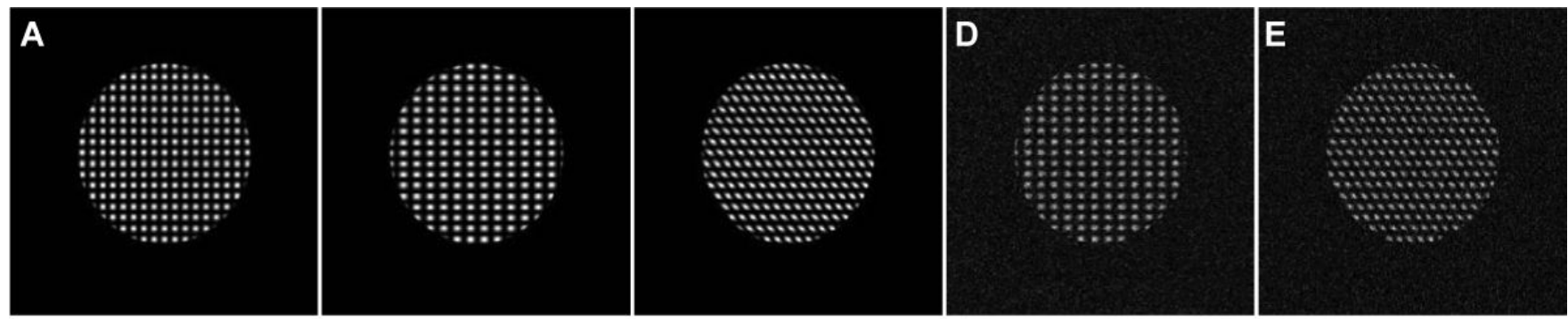

$\mathbf{F}$

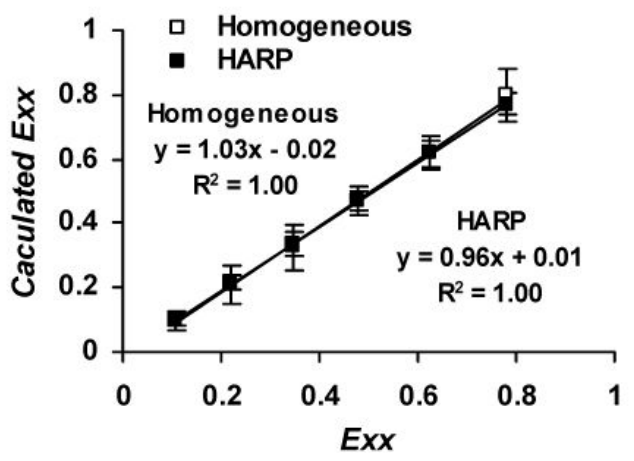

H

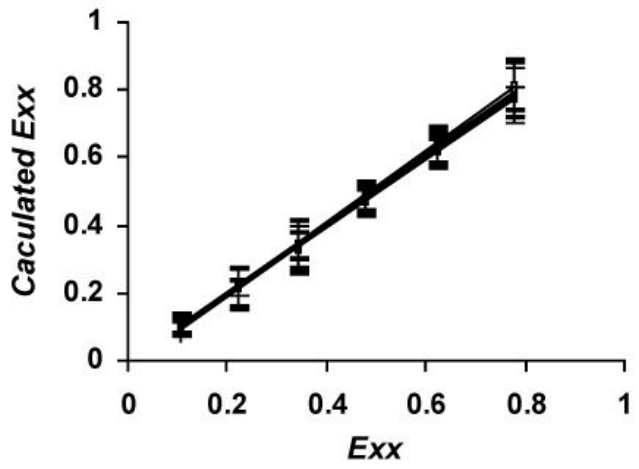

G

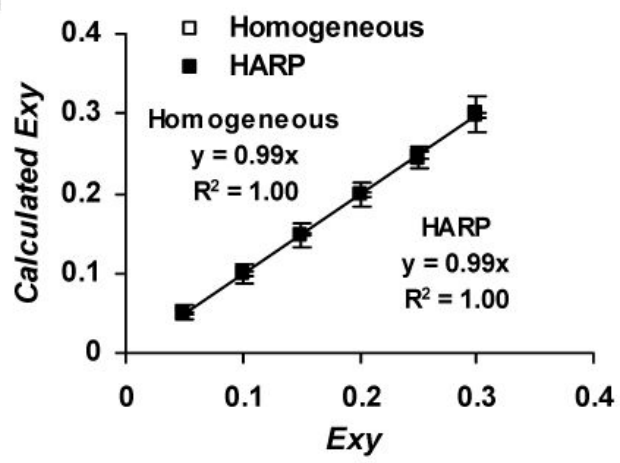

I

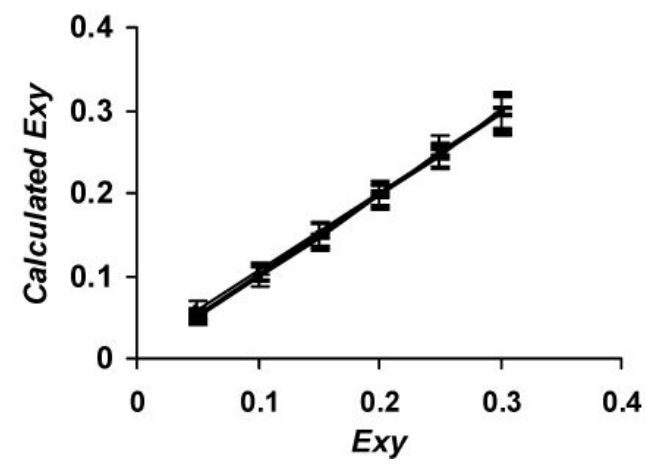

Figure 3.

Validation of strain calculation with simulated images. A. Reference image. B. Deformed image by stretch strain in horizontal direction $\left(E_{x x}=0.345\right)$ without noise. C. Deformed image by shear strain $\left(E_{x y}=0.3\right)$ without noise. D. Deformed image by stretch strain in horizontal direction $\left(E_{x x}=0.345\right)$ with $50 \%$ noise. E. Deformed image by shear strain $\left(E_{x y}=0.3\right)$ with $50 \%$ noise. F. Correlations of calculated stretch strains without noise. G. Correlations of calculated shear strains without noise. H. Correlations of calculated stretch strains with noise ranging from $20 \%$ to $100 \%$. I. Correlations of calculated shear strains with noise ranging from $20 \%$ to $100 \%$. 

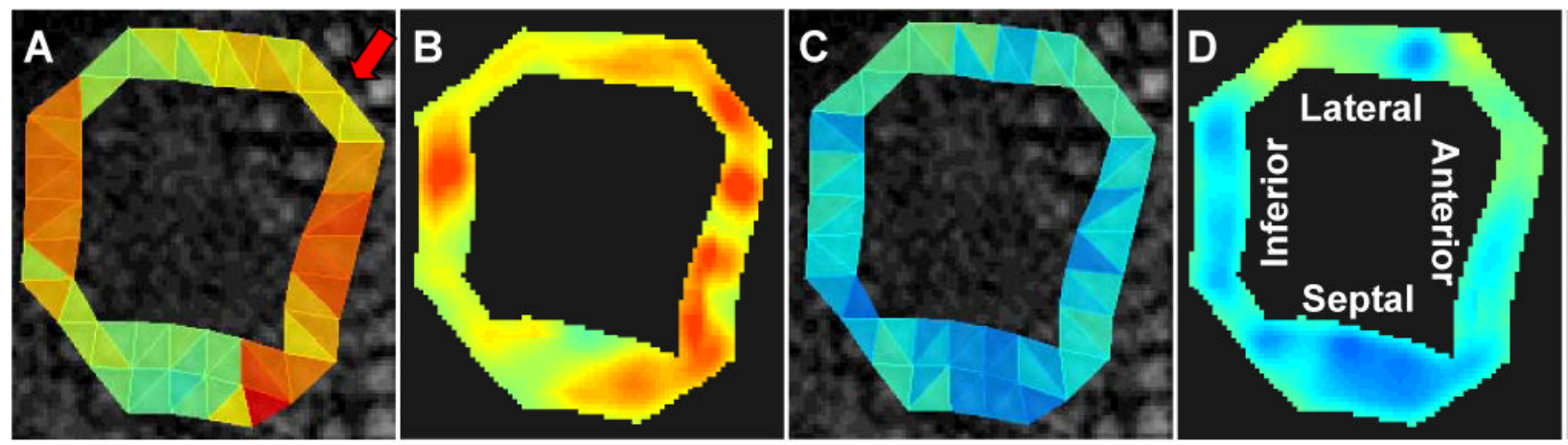

0.5
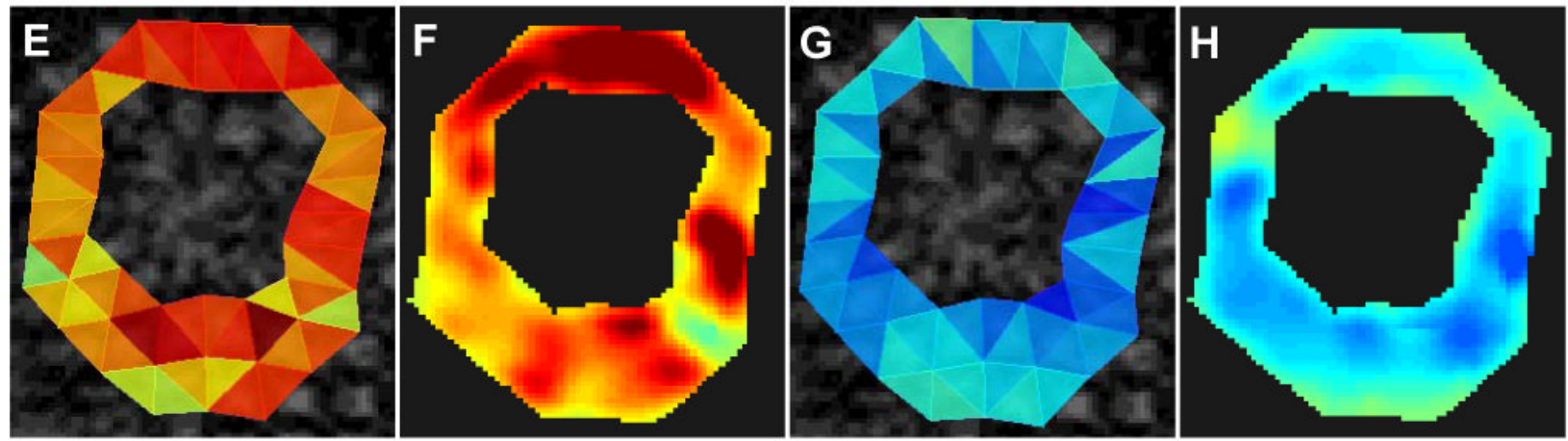

$-0.5$

\section{$E_{1}$ strain}

\section{$E_{2}$ strain}

Figure 4.

Representative strain maps from an infarct heart (top panels) and a control heart (bottom panels) at apical level. Red color indicates stretch and blue color indicates shortening. The red arrow indicates infarct region. A and $\mathrm{E}_{1} \mathrm{E}_{1}$ maps by homogeneous analysis. B and $\mathrm{F}$. $\mathrm{E}_{1}$ maps by HARP analysis. $C$ and $G$. $E_{2}$ maps by homogeneous analysis. D and H. $E_{2}$ maps by HARP analysis. 

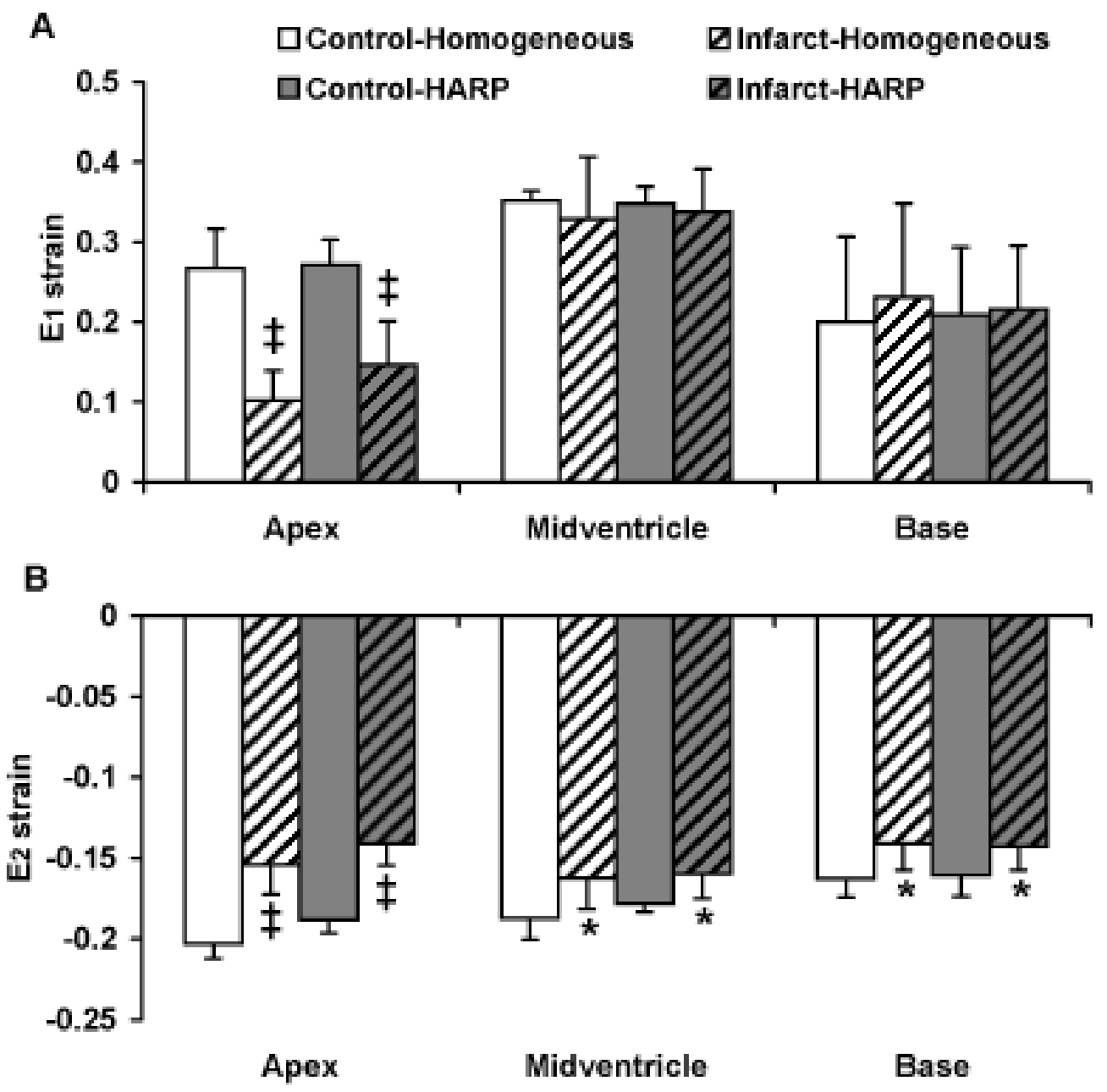

Figure 5.

Maximal stretch (A) and maximal shortening (B) assessed by homogeneous (white bars) and HARP analysis (gray bars) at apex, midventricle and base. ${ }^{*} \mathrm{p}<0.05$ vs control, ${ }^{\star} \mathrm{p}<0.01$ vs control. 
A

Maximal Stretch (E1 strain )

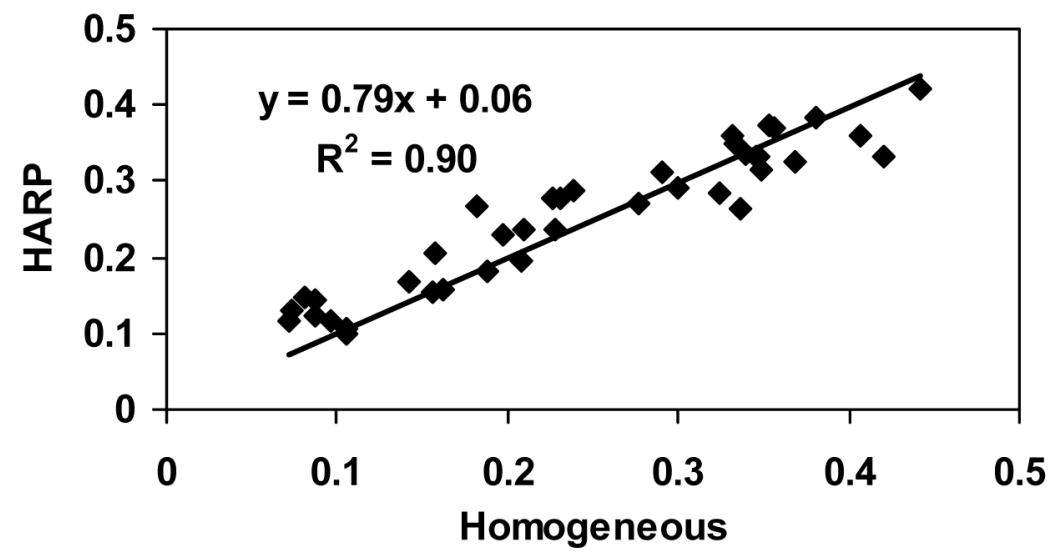

B

Maximal Shortening (E2 strain)

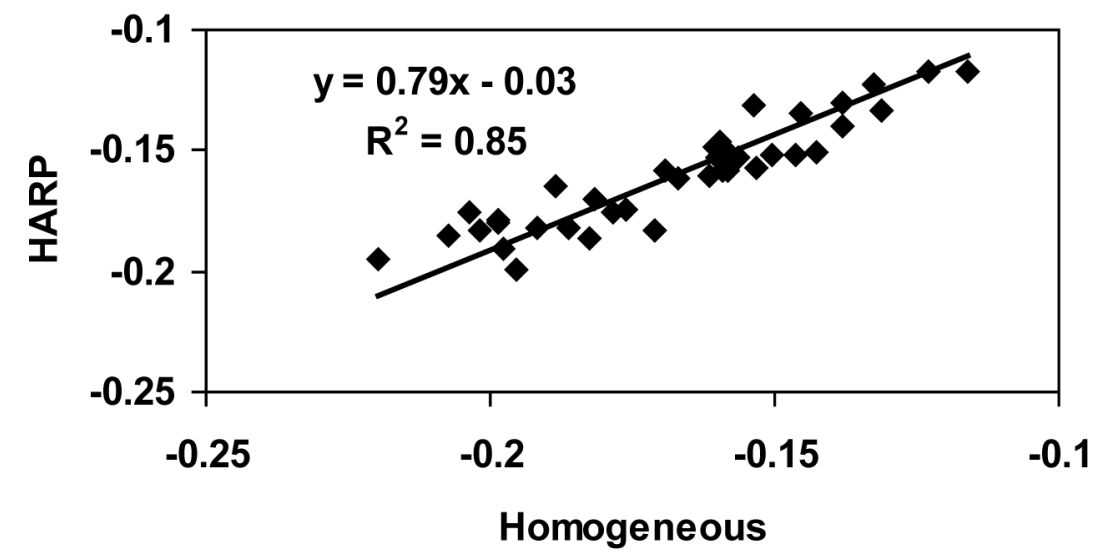

Figure 6.

Correlations between myocardial strains as assessed by HARP (y-axis) and homogeneous method (x-axis) for maximal stretch (A) and maximal shortening (B). 

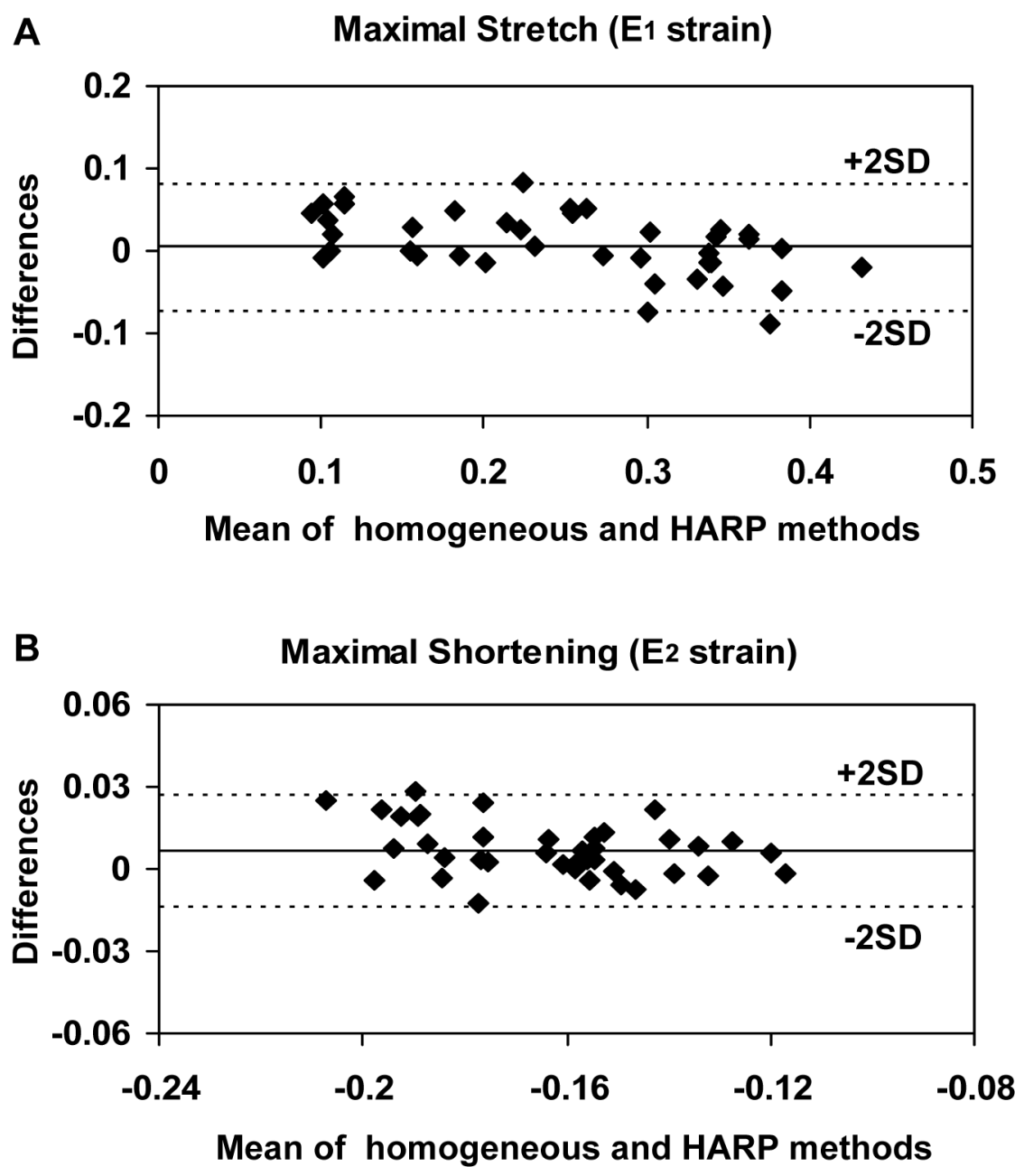

Figure 7.

Bland-Alman plots for the comparison of myocardial maximal stretch (A) and maximal shortening (B). 

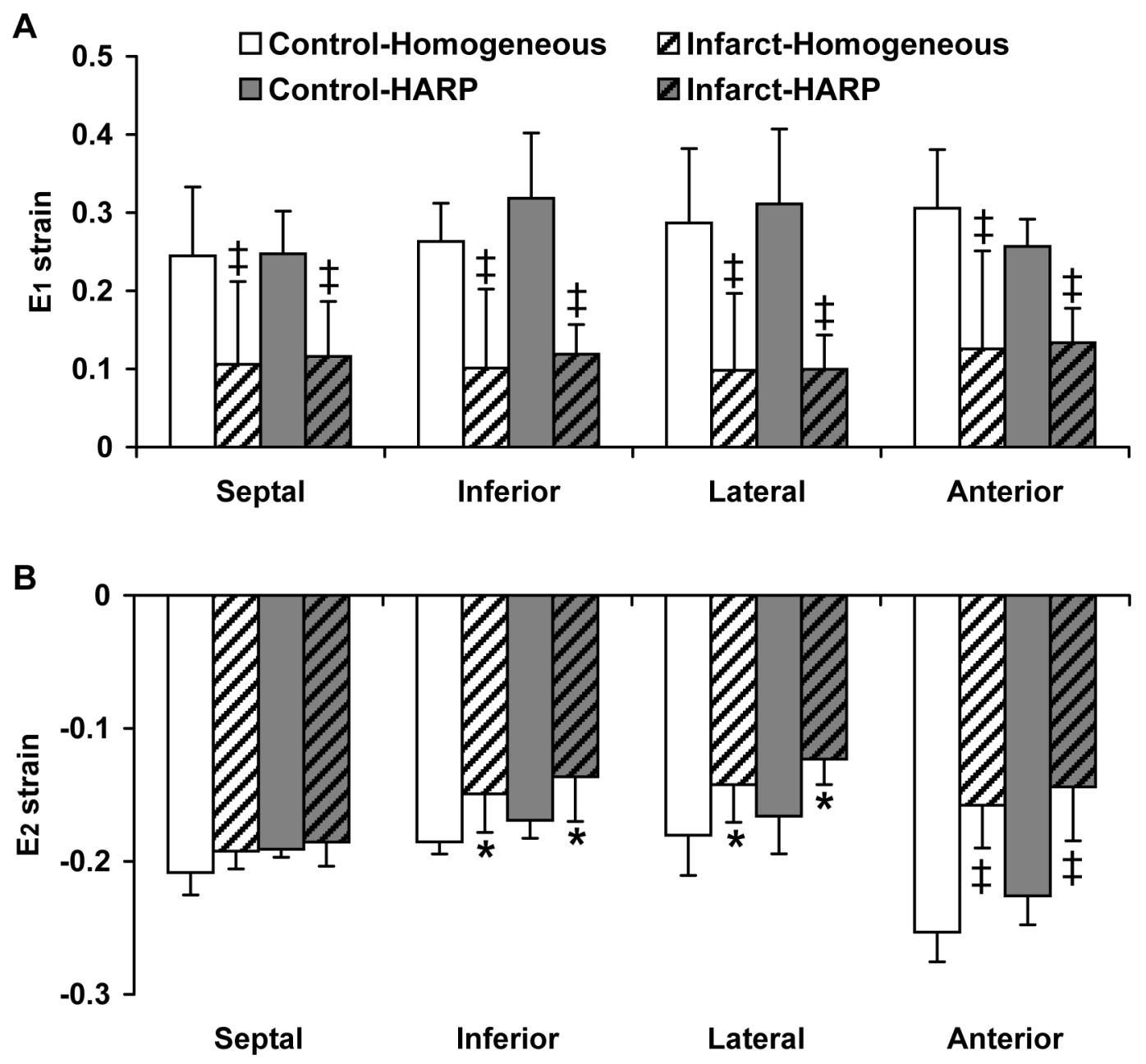

Figure 8.

Maximal stretch (A) and maximal shortening (B) assessed by homogeneous (white bars) and HARP analysis (gray bars) in segmented regions at apex. ${ }^{*} \mathrm{p}<0.05$ vs control, ${ }^{\ddagger} \mathrm{p}<0.01$ vs control. 

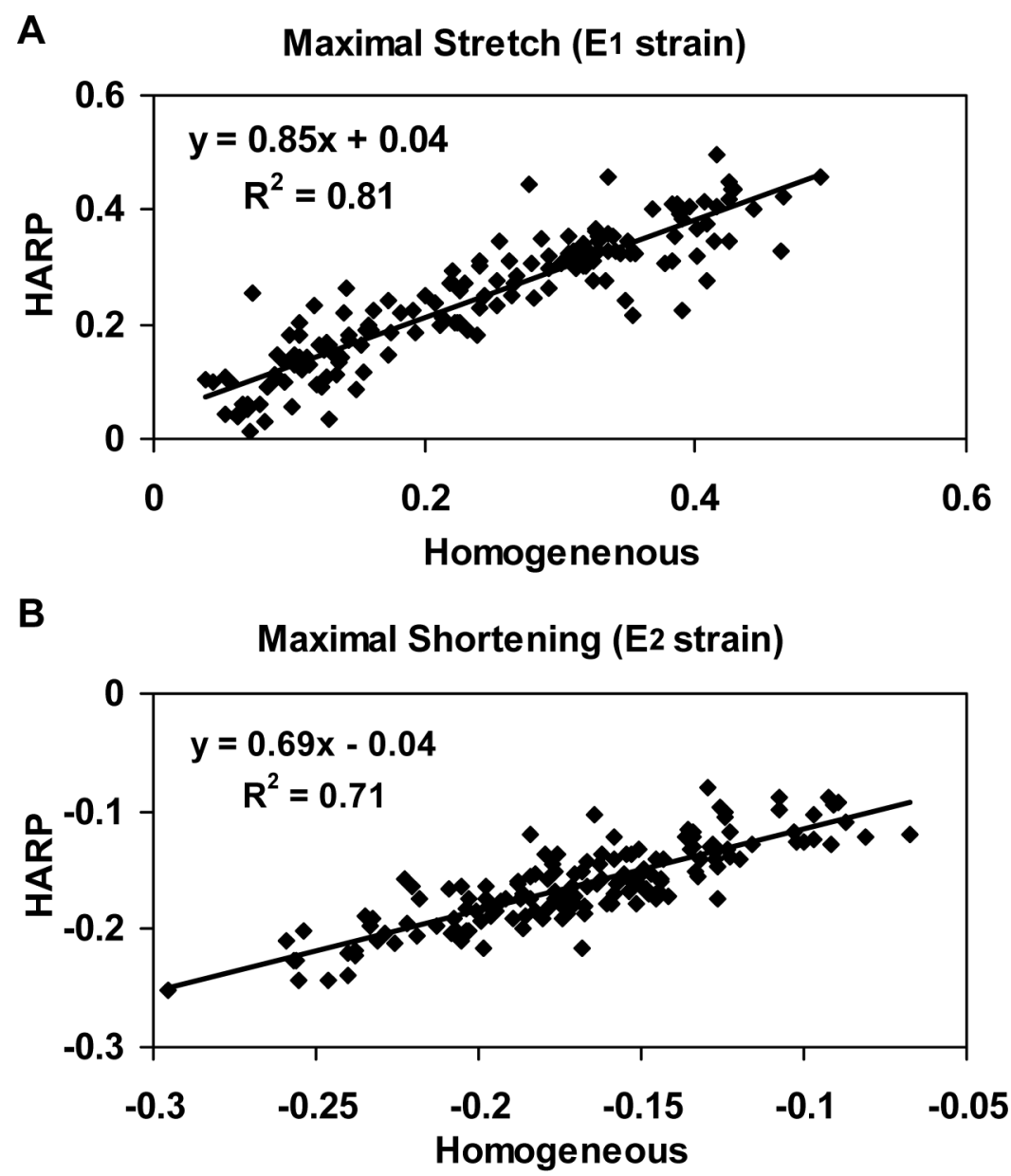

Figure 9.

Correlations between segmented myocardial strains as assessed by HARP (y-axis) and homogeneous method (x-axis) for maximal stretch (A) and maximal shortening (B). 


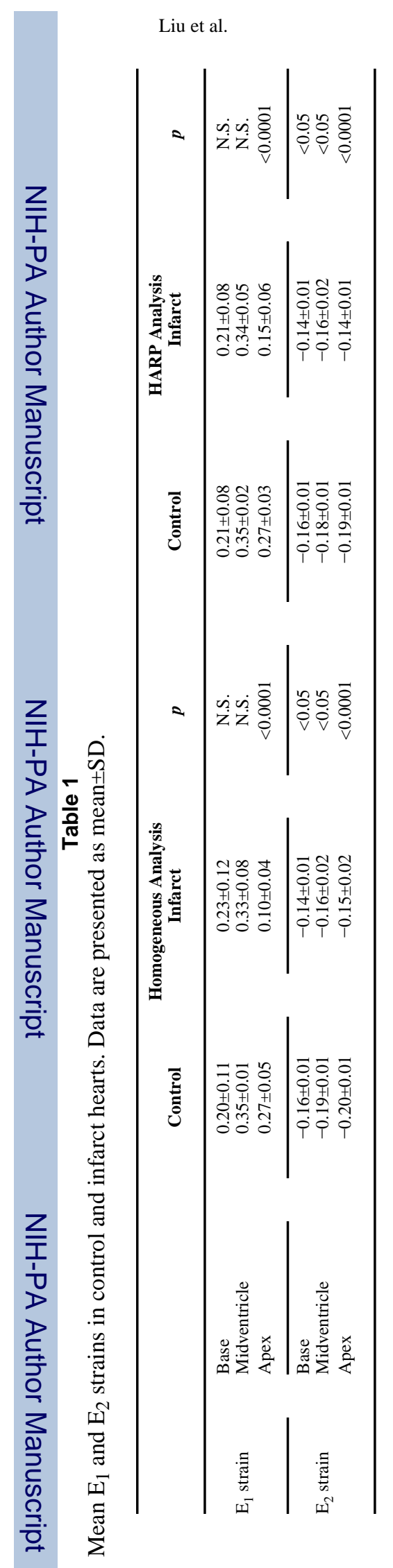

J Biomech Eng. Author manuscript; available in PMC 2008 May 30. 